\title{
Evaluation of Upper and Lower Pharyngeal Airways in Normodivergent Class I and II Malocclusions in a Group of Pakistani Patients
}

\author{
Omair Majeed ${ }^{1}$ \\ BDS \\ Tabassum Ahsan Quadeer ${ }^{2}$ \\ Maria Habib ${ }^{3}$ \\ BDS, FCPS \\ Maazia Jawaid ${ }^{4}$ \\ BDS \\ Rafia Mujahid 5 \\ BDS \\ BDS
}

\begin{abstract}
:
INTRODUCTION: Tooth wear is a global problem that involves an irreversible, non-carious loss of dental hard tissues due to repetitive physical contacts or chemical dissolution. The objective of the study was to determine the frequency and influential factors of tooth wear among patients visiting AKUH dental clinic.

METHODOLOGY: Male and female patients in the age range of 20-70 years, presenting to Aga Khan University Hospital with symptomatic or asymptomatic tooth wear in their permanent dentition were included in the study. Clinical examination included tooth wear assessment of all incisors and first molars using Smith and Knight Tooth Wear Index. All findings were recorded into the examination proforma. Data was analyzed and influential factors (such as bruxism, lack of canine guidance and TMJ clicking etc.) of the tooth wear were determined by using Chi square test.

RESULTS: There were 114 subjects, 64 males and 50 females. The mild, moderate, moderately severe and severe tooth wear was observed in $23 \%, 36 \%, 28 \%$ and $12 \%$ of the subjects, respectively. Both genders had similar tooth wear scores. Among molars, occlusal surface was affected more with the wear compared to buccal/ lingual surface. Incisors exhibited more tooth wear on the incisal surface compared to labia or palatal/lingual surfaces.
\end{abstract}

CONCLUSIONS: Age more than 45 years and presence of TMJ clicking was found to be significantly associated with the tooth wear in incisors and molars. The occlusal and incisal surfaces among bruxers were affected more with tooth wear compared to their other tooth surfaces.

KEYWORDS: Tooth wear; tooth attrition; tooth abrasion; tooth erosion.

HOW TO CITE: Majeed O, Quadeer TA, Habib M, Jawaid M, Mujahid R . Evaluation of Upper and Lower Pharyngeal Airways in Normodivergent Class I and II Malocclusions in a Group of Pakistani Patients. J Pak Dent Assoc 2017; 26(1): $22-25$

Received: 18 December 2016, Accepted: 27 February 2017

\section{INTRODUCTION}

$\mathrm{T}$ The growth and development of maxillofacial structures is influenced greatly by normal respiratory activity as a result of which significant relationships exist between craniofacial and pharyngeal structures. ${ }^{1}$

\footnotetext{
${ }^{1,3 \& 4}$ Senior Lecturers, Department of Orthodontics, Bahria University Medical and Dental College, Karachi, Pakistan

${ }^{2}$ Assistant Professor and Head of Orthodontics Department, Bahria University Medical and Dental College, Karachi, Pakistan

${ }^{5}$ Registrar, Department of Orthodontics, Bahria University Medical and Dental College, Karachi, Pakistan

Corresponding author: "Dr. Omair Majeed" < dromairis@hotmail.com >
}

Heredity has a big impact on the development of the human face and the airway while environment seems to play an important role in nasal obstruction. ${ }^{1,2}$

The values for normal upper airway space and lower airway space are $15-20 \mathrm{~mm}$ and $11-14 \mathrm{~mm}$ respectively. Skeletal features such as maxillary and mandibular retrusion and vertical maxillary excess in hyperdivergent patients may lead to narrower anteroposterior dimensions of the airway. ${ }^{2,3}$

Position of the tongue is affected by the anteroposterior position of the mandible because the genioglossus muscle of the tongue is connected to the inner surface of the anterior mandible. It has been observed that the size of the 
Pharyngeal Air Space is different among subjects with a normal, retruded or prognathic mandible. ${ }^{4}$ Pharyngeal air space diameter is different in different malocclusions and OSA is associated with narrow pharyngeal airspace and breathing problems.

The nasopharyngeal skeleton may change from adulthood to older ages and this change has an impact on respiratory activity. ${ }^{5}$

Factors that can hamper breathing and can indirectly affect craniofacial development include allergies, such as rhinitis, asthma, environmental irritants, enlarged adenoids, enlarged tonsils and viral infections. Nasal obstruction can also be caused by inferior turbinate hypertrophy leading to mouth breathing, obstructive sleep apnea as well as excessive daytime sleepiness. ${ }^{6}$

Mouth breathing has been associated with many unfavorable consequences which include excessively long tapered face form, increased lower face height, and narrow maxillary arch form. The mandible goes downward and backward resulting in an increase in lower facial height as well as cranial extension because of the backward tipping of the head.

In our study, we plan to find out if any variation exists in pharyngeal airway spaces in patents with different anteroposterior skeletal patterns.

\section{METHODOLOGY}

This was a cross sectional pilot study carried out in the Orthodontic department, Bahria University Medical and Dental college, Karachi from $1^{\text {st }}$ April, 2016 to $31^{\text {st }}$ May, 2016. The record of 60 patients was selected from the orthodontic record of the department. The inclusion criteria were a Pakistani ethnicity, an age range of 12-20 years, Class I or Class II malocclusions based on ANB and high quality cephalograms. The exclusion criteria included patients with previous orthodontic treatment, syndromic patients, history of mouth breathing, history of adenoidectomy or tonsillectomy as well as patients with pharyngeal pathologies. The age of each patient was recorded from the orthodontic record file. The pretreatment radiographs of the subjects were traced manually on acetate paper by the principal investigator. To decrease the examiner bias, 20 radiographs were randomly selected and retraced by a second investigator to check for any difference in calculated values. Fig. (1) shows the landmarks, linear and angular measurements that were drawn and recorded on the tracing of each subject.
All patients who were included in the study were divided into Class I and II based on ANB (Skeletal class I: $2-4^{\circ}$ and Class II: $>4^{\circ}$ ). Their cephalometric radiographs were traced and upper and lower pharyngeal airways were measured manually on an acetatetracing paper with pencil. The following are some cephalometric points, lines and planes used in this study:

PSP: The PNS part of the soft palate.

PPW: The posterior pharyngeal wall.

HB: The superior margin of the body of the hyoid bone

RL1: Horizontal reference line passing through $S$ point $7^{\circ}$ to SN plan.

RL2: Vertical reference line passing through $S$ point perpendicular to $\mathrm{R} 1$.

We used the method of pharyngeal space measurement given by McNamara. The pharyngeal airway space was measured at two levels; upper and lower. First a reference lines RL1and RL2 planes were drawn. Upper Pharyngeal Air-Way width (UPAW) is the distance from PSP which is the PNS part of the soft palate to the posterior pharyngeal wall perpendicular to the reference line and the Lower Pharyngeal Airway width (LPAW) the distance between anterior and posterior pharyngeal wall perpendicular to the reference line at the level of the superior margin of the body of the hyoid bone.

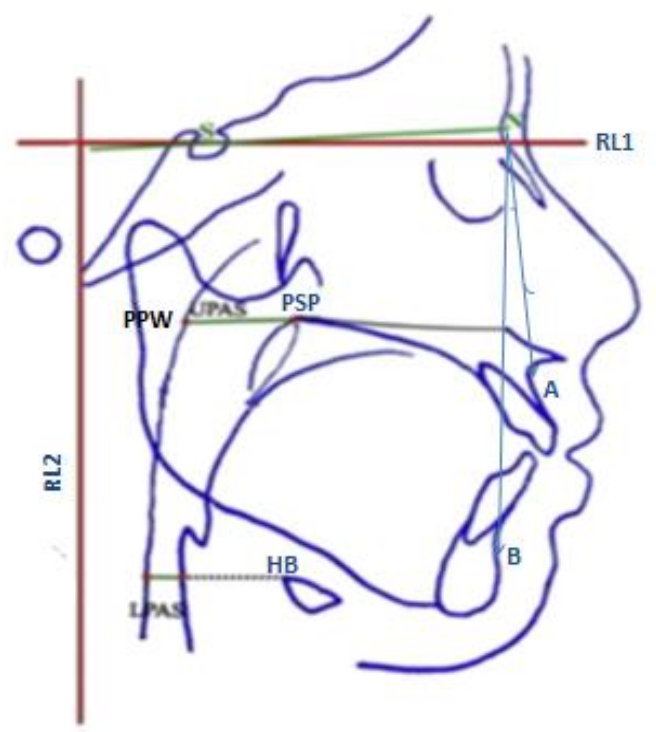

Fig. (1).

The statistical analysis was performed by using SPSS version 17 .The significance level was set at 0.05 . Independent 
sample t test was employed to evaluate the difference between upper and lower pharyngeal spaces in Class I \& II malocclusions.

\section{RESULTS}

The study sample comprised a total of 60 subjects (30 Class I and 30 Class II). The means and standard deviations of the upper and lower pharyngeal airways was determined for the sample. There was no statistically significant difference observed in the mean and standard deviation scores for upper and lower airway spaces between Class I and II malocclusions as shown in Table 2.

Table 1. Descriptive analysis of independent sample $t$ test.

\begin{tabular}{|c|c|c|c|}
\hline Malocclusion & Air way Space & Mean & SD \\
\hline \multirow{2}{*}{ Class I } & Upper Air way & 22.34 & 4.3 \\
\cline { 2 - 4 } & Lower Airway & 12.41 & 2.32 \\
\hline \multirow{2}{*}{ Class II } & Upper Air way & 21.10 & 3.68 \\
\cline { 2 - 4 } & Lower Airway & 12.03 & 2.07 \\
\hline
\end{tabular}

Table 2. Comparing airway spaces in Class I II malocclusions (Independent sample $\mathrm{t}$ test).

\begin{tabular}{|c|c|c|}
\hline Class I & Class II & $\boldsymbol{P}$ \\
\hline Upper Air way & Upper Air way & 0.24 \\
\hline Lower Airway & Lower Airway & 0.50 \\
\hline
\end{tabular}

\section{DISCUSSION}

The ability of the orthodontist to visualize beneath soft tissue and evaluate structures that contribute to facial development has been provided by cephalometric studies. ${ }^{2}$ Individuals exhibiting Class I, II and III malocclusions, when compared, have shown significant differences for the upper and lower airways. ${ }^{6}$ Abnormal development of the maxillofacial area can be caused by adenoid or tonsillar obstruction and surgical treatment of either problem leads to improvement of the airway. ${ }^{1}$

In this study, we evaluated the effect of skeletal malocclusions on the upper and lower airway. As mouth breathing can lead to reduced airway dimensions, subjects with normal nasal breathing were selected for the study. Our results showed that there was no statistically significant difference in both the upper and lower airway structures in both Class I and II malocclusions. The mean value for upper airway in Class I and II was $22.3 \mathrm{~mm}$ and $21.1 \mathrm{~mm}$ whilst the mean for lower airway in Class I and II was $12.4 \mathrm{~mm}$ and $12 \mathrm{~mm}$ respectively.

Memon et al in their study showed that there was no difference in upper and lower airway in normodivergent and hypodivergent facial patterns and concluded that sagittal malocclusion has no influence on upper pharyngeal width but hyperdivergent facial patterns were an exception. ${ }^{7}$ As our study did not include the hyperdivergent cases, our results did not come out as significant.

Similar results were found in the study of Soheilifar ${ }^{8}$, who showed that airway dimensions were not significantly different between patients with skeletal Class I and Class II and that the ANB difference did not have a direct influence on airway dimensions and the dimensions of soft palate.

Kirjavainen reported that Class II malocclusion is associated with narrow upper airway without retrognathia, ${ }^{9}$ however our study did not show this result. One reason for this could be our limited sample size, we might get significant results once the sample size is calculated and increased.

Freitas et al in their study compared the airway of Class I and II malocclusion with normal and high growth patterns and found no difference in the upper and lower airway in Class I and II normodivergent facial patterns. However the result was quite different when Class I and II hyperdivergent facial patterns were studied showing marked difference in the upper airways with Class II having narrow upper airway. The upper pharyngeal airway width is not influenced by type of malocclusion while lower pharyngeal airway width is not influenced by malocclusion type and growth pattern. ${ }^{2,10,11 .}$

In a study by Reddy et al, ${ }^{6}$ a larger pharyngeal size was associated with class II normodivergent pattern than class I normodivergent pattern which is in contrast to our study. This study also emphasizethe fact that the comparison of upper and lower airways in a normal and vertical growth pattern in a class I, II and III skeletal pattern showed no significant results when done independently.

Jain et al in their study showed that oropharynx is significantly different among the three skeletal malocclusion groups by being widest in skeletal Class III malocclusion group and in both males as well as in females followed by Class I and then being narrow in Class II. ${ }^{12}$

Iqbal reported no statistically significant difference in upper airway width between hypodivergent and normodivergent facial pattern of skeletal Class I and II subjects. $^{13}$ 
It is suggested that more accurate conclusions can be derived if an adequate subject number; bigger samples as well as factors like divergence of the face are also taken into consideration for the study.

\section{CONCLUSION}

There was no impact of skeletal malocclusion on the upper and lower airways in normodivergent patients in our study, as both were found to be within normal limits.

\section{REFERENCES}

1. Elwareth AA, Yousif AE. Evaluation of upper and lower pharyngeal airway in hypo and hyper divergent Class I, II and III malocclusions in a group of Egyptian patients. Tanta Den J. 2015;12: 265-76.

2. Batool I, Shaheed M, Rizvi SAA, Abbas A. Comparison of upper and lower pharyngeal airway space in Class II high and low angle cases. Pak Oral Dent J 2010;30:8185 .

3. McNamara JA Jr. A method of cephalometric evaluation. Am J Orthod 1984; 86: 449-69.

4. Shaikh SY, Malik AM, Khalid O, Mahnoor M. Comparison of upper pharyngeal airway space in Class II and Class III malocclusion cases. Pak Orthod J 2014;6: 2-6.

5. Ashwin KV, Kumar A, Subbiah S, Senkutvan RS. Pharyngeal Airway Dimension in Different Types of Malocclusion. Int J Dent Sci Res 2014;2:7-11.
6. Reddy R, Chunduri R, Thomas M, GAnapathy K , Shrikant S, Chandrashehkar MH. Upper and lower pharyngeal airways in subjects with skeletal Class I, Class II and Class III malocclusions and different growth patterns- A Cephalometric study. INt J Contemp Dent 2011;25:12-18.

7. Memon S, Fida M, Shaikh A. Comparison of different craniofacial patterns with pharyngeal widths. J Coll Physicians Surg Pak 2012;22: 302-6.

8. Soheilifar S, Soheilifar S, Soheilifar S. Upper airway dimensions in patients with class II and Class I skeletal malocclusions. Avicenna J Dent Res. 2014; 6 (2): e23300.

9. Kirjavainen M, Kirjavainen T. Upper airway dimensions in Class II malocclusions. Angle Orthod. 2007; 77: 1046-53.

10. Zhong Z, Tang Z, Gao X, Zeng XL. A Comparison Study of Upper Airway among Different Skeletal Craniofacial Patterns in Nonsnoring Chinese Children. Angle Orthod. 2010; 80: 267-74.

11. Freitas MR, Alcazar NMPV, Janson G, Freitas KMS, Henriques JFC. Upper and lower pharyngeal airways in subjects with Class I and Class II malocclusions and different growth patterns. Am J Orthod Dentofacial Orthop 2006; 130: 742-5.

12. Jain S, Raghav P, Misra V, Reddy CM, Singh S, Agarwal S. Assessment of Upper and Lower Pharyngeal Airway Width in Skeletal Class I, II and III Malocclusions. J Ind Orthod Soc 2014; 48: 446-53.

13. Iqbal N, Rasool G, Alam T, Hussain U, Shah SS. Comparison of different craniofacial patterns with pharyngeal widths. J Khyber Coll Dent 2015; 6:20-24.. 